\title{
A Survey on hierarchical cluster-based energy efficient routing protocol for wireless sensor networks
}

\author{
Humera Khan* \\ M.Tech Scholar, Department of Computer Science and Engineering, SRM University NCR Campus, Modinagar, Ghaziabad (U.P) \\ *Corresponding author E-mail: hammu1215@gmail.com
}

\begin{abstract}
Wireless Sensor Network is a widely growing field and it comprises of tiny sensor nodes. These sensor nodes are distributed in the environment spatially. They are capable of sensing the environment, gathering the information and processing it. Each sensor node collaborate with other sensor nodes for processing the information. Sensor nodes have very limited resources available for their operation. For the purpose of consuming resources in an efficient way several routing algorithms are employed. Here the focus is mainly on hierarchical cluster based routing techniques. In this paper we provide an introduction for wireless sensor network, the requirement for reduction in energy consumption of sensor nodes and some of the already existing energy efficient routing protocols of wireless sensor network.
\end{abstract}

Keywords: Wireless Sensor Networks, Clustering, Routing, Hierarchical Clustering, Energy Efficient Routing, etc.

\section{Introduction}

Wireless networks comprises of computer networks that does not require any kind of cable connection. With the introduction wireless network in the enterprises the costly process of using cables into buildings and for connection between different equipments is been avoided. The communication in wireless systems take place through radio waves as radio waves are the basis of the system and their implementation takes place at the physical level of network structure. Nodes are not connected by any physical medium for communication. In spite they use wireless mediums such as air / atmosphere for data transmission from one node to another. Mediums like Radio Waves Communication, Microwave Communication Satellite Communication, etc. are most commonly used medium for wireless transmission.

This network includes a very massive number of ambient, autonomic, lesser power devices which are sensors and they are dispersed. They are associated to aggregate, operate, and assign data to the sink. Secondly it also composed the capabilities like calculation \& processing [3].

The decision of sensor nodes for collection of data is based on its overlook and on partial a-priori information. It is very essential for sensor nodes to be fault tolerant and power house as these sensor nodes are deployed in rough environment. The event should be diagnose rapidly and the estimations are done alongside in wireless sensor network.

Here problem of design of optimal parallel distributed computational architecture is investigated. In case of distributed system the components which are located on networked computers communicate with each other and creates coordination by flowing messages to perform the specified task accordingly. Similarly defined computational model is required for performing distributed computation on distributed nodes which are connected The major characteristics of WSN includes:

- Power adaptability in WSN

- Ease of adoption

- WSN impartiality and acceptance

- Movableness over the network. A computational model is a description which gives description about a particular type of computational process[8]. This paper assumes that no memory computational model of sensor nodes are used as architecture for primitive recursive functions. The meaning of no memory computational model represent that the sensor node just contain the registers for storing two values. On reception of any value from sensor node, further calculations proceed between the receipt value and the one it has calculated and finally passes on the report to other nodes.

Here sensor comprises of Radio Transceiver which transmits the information (Transmitter) and receives information (Receiver). An Antenna for providing connectivity to network. Micro Controller for purpose of controlling sensor operation. An electronic circuit which serves interface between different sensors and finally a battery which is the source of energy as each element requires energy for operations. They usually composed of Processing Unit with bound computational capabilities, defined memory and confined energy [3].

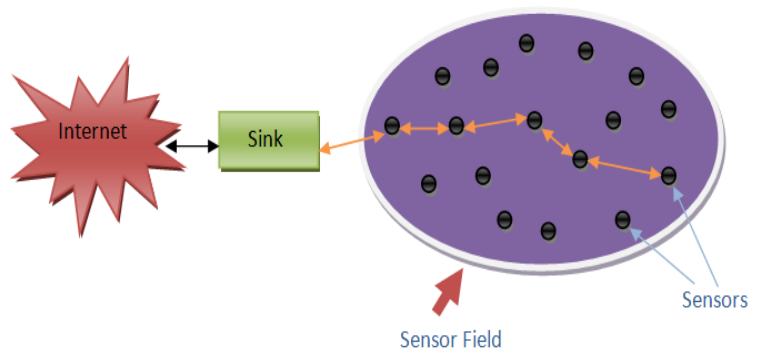

Fig.1: Sensor Network

- $\quad$ Reliability

- Network topology is charismatic

- Revelation collapse

- Assortment among the sensor nodes

- Scalability to large scale of deployment 
- Endowment of the nodes to confront worst conditions of the environment

- Unattended operation

- Ability to survive node failure

\section{Energy in Wireless Sensor Networks}

Sensor Nodes acquire energy and whenever they transfer information or during the reception of information among sensors this energy is exploited. This transfer of data requires energy usage. Therefore, reducing energy consumption of sensor nodes and prolonging its lifetime has become the core problem of wireless sensor network. To establish an energy efficient routing algorithm in WSN is the main objective of the effort.

As sensor nodes operate on battery and they have very limited energy resources therefore the finest way to actuate span of
WSN lifetime . As the WSNs node typically stay at hostile environment where recharging and replacing of battery is not very easy, which limits the lifetime of the entire sensor network. Therefore energy optimization is very complex in sensor networks because as the energy consumption will be reduced their will be increment in lifetime of network. As the awareness in the energy consumption field introduces in its architecture it can be acquire by. It provides the assurance about energy awareness of the network.

\section{Routing Protocols in WSN}

In case of wireless sensor networks the routing is distinct in various ways from conventional routing of fixed networks. For wireless networks multiple routing algorithms came into existence and are categorised as:

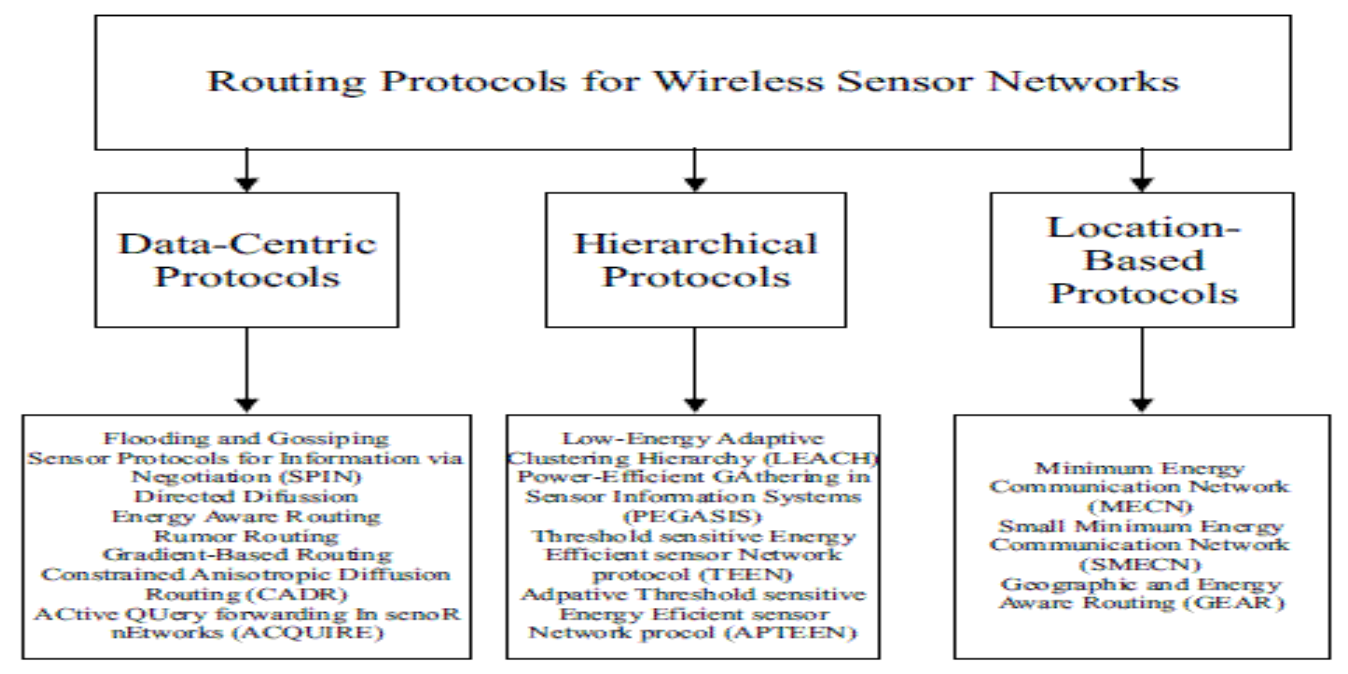

Fig.2: Routing Protocol Hierarchy Representation of Sensor Network

This work gives a review over energy adequate Hierarchical Routing Protocols.

\section{A. Hierarchical Routing Protocols}

These protocols are energy adequate communication protocols and they are utilized by the sensor nodes for data transmission to the to the base station (sink). Main goal of these protocols is to productively use the energy consumption of sensor node along with relating them in multi-hop communication inside a specific cluster. By executing data aggregation and data fusion it will reduce the number of transmitted message to the base station (sink). Thus reducing the consumption of energy.

\section{B. Low-Energy Adaptive Clustering Hierarchy (LEACH)}

LEACH is an energy adequate hierarchical routing protocol of WSN that came into existence for reduction of power exhaustion. The reason behind the existence is the accession in

$$
\mathrm{T}(\mathrm{n})=\frac{P}{1-P *\left(\operatorname{rmod} \frac{1}{p}\right)} \quad \text { if } \mathrm{n} \in \mathrm{G}
$$

where $\mathrm{P}$ represent the desired percentage of cluster head, $\mathrm{r}$ stands for current round and $\mathrm{G}$ belongs to the set of nodes that have not been elected as cluster head in last rounds. (ii) a steady-state phase for collecting data, compressing it and transmitting it to the sink. The energy usage is depreciated by (a) the overheads of conveyance is depreciated and (b) non $\mathrm{CH}$ nodes which are inactive, they are turned off. Furthermore, the idea of dynamic clustering brings extra burden, e.g. cluster head changes, advertisements etc., which may reduce the lifespan of the network by the introduction of clustering approach in WSN[1].

The vast network which comprises of several nodes split into clusters, whose architecture comprises cooperation and control. Each cluster comprises of a cluster head $(\mathrm{CH})$ for data transmission. The information is transferred between $\mathrm{CH}$ and $\mathrm{BS}$ directly by single hop. This clustering will not only reduce the amount of data to be transmitted to the base station(BS), thus reducing the energy consumption of nodes as well as to create routing and conclusion allotment more expandable and robust. LEACH uses an unconsidered adjustment for the distribution of $\mathrm{CH}$ position, $\mathrm{CH}$ selection is arbitrary rather than electing in firm manner, in case for all nodes to annex similar opportunity to act as cluster head $(\mathrm{CH})$.

The LEACH operation is further classified as (i) Initially a setup phase which is responsible for dividing the whole network into clusters, all the sensor nodes are unconsidered and ascribe a unconsidered number between 0 and 1 . After the calculation of threshold, if unconsidered number threshold $\mathrm{T}(\mathrm{n})$ is greater than produced number it would be casted as $\mathrm{CHs}$. The calculation for Threshold value $T(n)$ is given as:

accumulation in energy consumption. LEACH clustering concludes in specific recurrence, but does not guarantee good $\mathrm{CH}$ dissemination as $\mathrm{CH}$ election is random and assumes uniform energy consumption for $\mathrm{CHs}$.

\section{Power-Efficient Gathering In Sensor Information Systems (PEGASIS)}

PEGASIS is an improved version of the LEACH protocol, that forms a chain of sensor nodes so that each node can transmit and receive data from a neighbour and only single node is selected from the chain which is responsible for transmitting data to the base station. All the sensors transmit data to its local neighbours 
in spite of sending it to the $\mathrm{CH}$ unlike LEACH. PEGASIS routing protocol, first comprises of construction phase, in which it assumption is that all the sensors contains knowledge about the entire network topology especially, the location of the sensors. After that it uses greedy approach. After the start of algorithm if any sensor node fails due to low battery power then that sensor node is isolated in the network, the chain is constructed again and utilizes the same greedy approach. In each round, a sensor node is selected randomly from the chain and it will transmit the collected data to the BS, thus reducing energy utilization as compared to LEACH

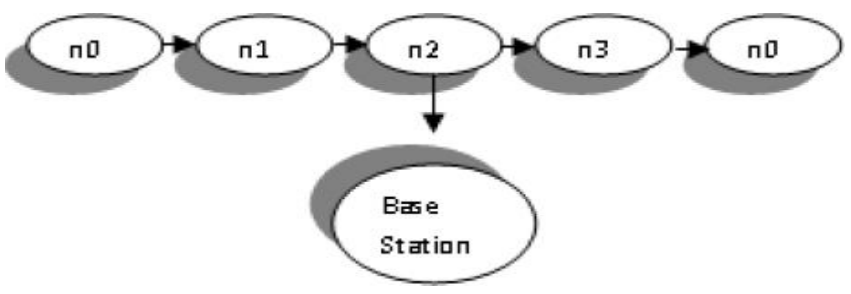

Fig.3: Clustering in PEGASIS

Simulation results of PEGASIS shows it will improve the lifetime of WSN by a factor of two in comparison to LEACH protocol. The improvement in the performance of PEGASIS is achieved by reducing overhead of $\mathrm{CH}$ election including reduction in number of transmissions and receptions.

Although the overheads due to cluster formations are avoided, still there is the requirement of dynamic changes in network topology of PEGASIS since the sensor nodes must have information regarding remaining energy status of its neighbours so as to identify which route to forward its data. Such topological adjustments when used in highly utilized networks they introduce high over head[7].

\section{Hybrid, Energy-Efficient Distributed Clustering (HEED)}

HEED is the modification of the basic scheme of LEACH as it fuses the residual energy and node degree or density as the parameters for the selection of cluster in case to accomplish power balancing. In HEED the election of cluster heads depend upon the amount of energy that is distributed in relation to a neighbouring node. It serves in multi-hop networks and employs an adaptive transmission power for inter-cluster communication [6].

HEED comprises of an architecture with four major goals namely (i) stretching network lifetime by distributing energy consumption, (ii) eliminating the clustering process within a constant number of iteration, (iii) reduction in control overhead and (iv) production of well distributed cluster heads and packed clusters across the network.

In HEED, the criteria for electing $\mathrm{CH}$ is based on two parameters, viz. Residual Energy and Intra-Cluster communication cost.

Communication Cost. The first parameter includes computing the probability of each node for becoming a cluster head and second parameter comprises of a function of node degree. The primary parameter is used for electing an initial set of $\mathrm{CHs}$ while the secondary parameter is avail as the breaker.

Simulation result comprises that HEED clustering stretches network lifetime in comparison to LEACH because in LEACH cluster head is elected on a random basis, which results in reduction of lifetime of sensor nodes. Then finally the $\mathrm{CHs}$ which are selected in HEED are approximately distributed across the network equally resulting in the extension of the lifetime of the nodes thus stabilizing the neighbourhood within the network. There is no requirement of special node capabilities like location awareness and it does not make assumptions about node distribution. Thus communication cost is minimized. However, the selection of clusters agrees with only a subset of parameters, which can possibly apply certain constraints on the system.

These techniques are appropriate for enhancing the lifetime of network in spite for the entire needs of WSN [6].

As in LEACH, a preliminary percentage of cluster heads Cprob is predefined in the network and it has no direct impact on the final cluster structure. In HEED, each sensor node has the probability $\mathrm{CH}$ prob of becoming a cluster head given as follows;

$$
\mathrm{CHprob}=\mathrm{Cprob} \frac{\text { Eresidual }}{\text { Emax }}
$$

\section{E. Threshold Sensitive Energy Efficient Sensor Network Protocol (TEEN)}

TEEN is another similar protocol, which is responsible for dividing sensor network into clusters and each cluster is headed by a $\mathrm{CH}$. In each cluster all the sensor nodes report the sensed information to their $\mathrm{CH}$. Reception of data occurs at $\mathrm{Ch}$ and level by level it is forwarded to the sink simultaneously. The, architecture of TEEN is based on a hierarchical groupings in which adjacent nodes form clusters. This process proceeds to the further levels until the sink is not encountered.

Applications in which teen has a very crucial impact includes employment where users have authority of trade-off between data certainty, energy adaptability and response time.

In TEEN data-centric method is used along witH hierarchical approach. Important features of TEEN is that energy consumption for message transmission is more as compared to data sensing, as a result it reduces energy consumption of sensor nodes and increment of network lifetime. Drawbacks report that TEEN is not suitable for sensing applications where timely reports are required [2].

\section{F. Adaptive Threshold Sensitive Energy Efficient Sensor Network Protocol (APTEEN)}

APTEEN is an another protocol as it is enhancement of TEEN its main objective was to succeed the imperfections. Its objective comprises of reciprocating time critical activities as well as aggregating the periodic data [3]. APTEEN permits the sensed information collected by sensor to be broadcasted according to the time. By accommodating the enhanced data to $\mathrm{CHs}$, it permits response to real time modification.

APTEEN has the similar network architecture as TEEN APTEEN ensures abatement in utilized energy gives increment in life span of network and the life span of the sensors also improves. APTEEN provide support to three different types of queries namely (i) historical query, for analyzing old data , (ii) one-time query, which is used for aggregating the current exemplifation of network; and (iii) persistent query, for monitoring an activity for a certain time duration [4]. 


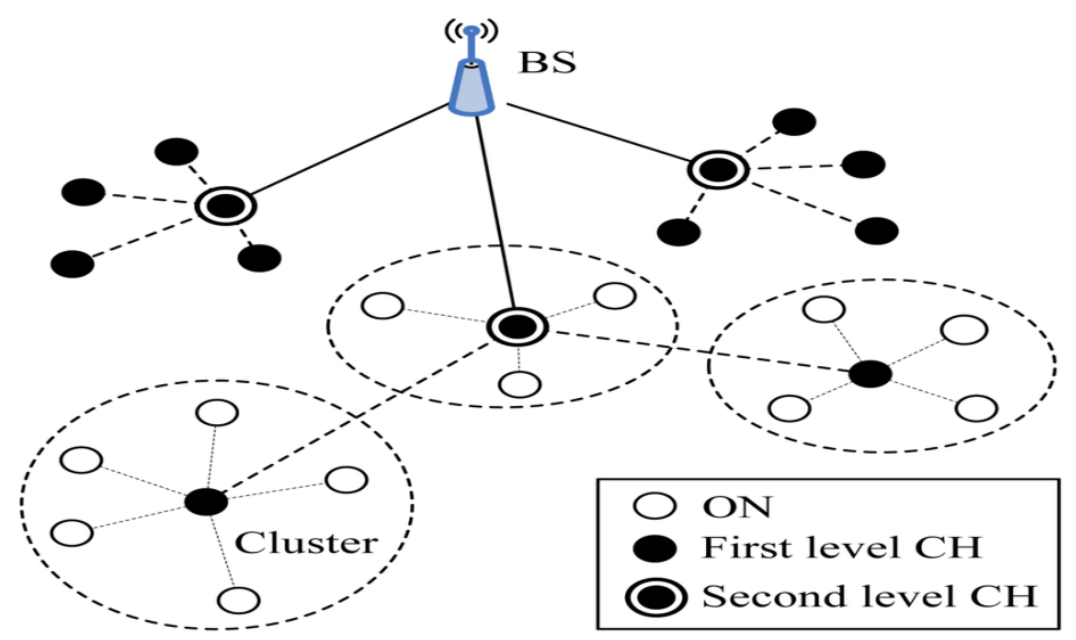

Fig.4: Clustering Representation in TEEN and APTEEN

\section{G. Distributed Weight - Based Energy - Efficient Hierarchical Clustering (DWEHC)}

It is some different clustering algorithm which is proposed for achieving a balanced cluster size and optimizing intra cluster topologies in WSNs. There are some a likenesses between DWEHC and HEED in addition to no estimations of network size and density, and taking into account residual energy in the process of cluster head $(\mathrm{CH})$ selection. But it assumes that sensor nodes are aware about the location of other sensor nodes and transmission is carried out for same fixed power levels. The cluster node which is at the farthest distance from the $\mathrm{CH}$, its cluster radius ( $R$ ) is fixed for the whole network. Firstly it runs seven iterations on each node, after that DWEHC introduces a multi hop intra-cluster structure which comprises of cluster head at the root and member nodes in a breadth - first order. Each cluster consist of multi levels of child node.s In this protocol each cluster accommodate some number of levels which is possessed by the cluster radius(R). It also requires the least energy path from any node within cluster to its cluster head $(\mathrm{CH})$ as the approximation of the size and network topology is null. The data reaches the $\mathrm{CH}$ as each node respond to its nearest parent and each parent respond to its own parent simultaneously. In this way data travels from each node to $\mathrm{CH}$. Intra-cluster communication requires Time division multiple access (TDMA) scheme and 802.11 protocol which is required by cluster head channel for information transferral to the base station [4].

\section{Models Used in Clustering Protocol}

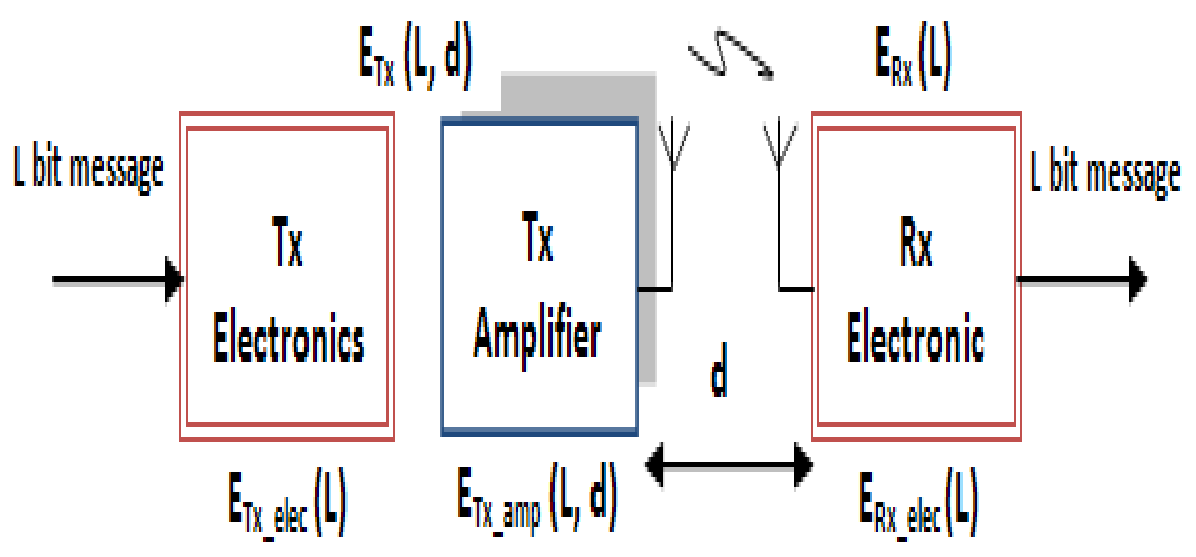

Fig.5: Radio Energy Dissipation Model
The system models which are used for energy dissipation in Wireless Sensor Network are following:

\subsection{Network Model}

The network model incorporates the environmental area which embrace the sensor nodes and base station.

This $\mathrm{N} \times \mathrm{N}$ region represent the region of network that incorporates the nodes with the basic assumption that sink is established at the centre. These nodes in the network environment sense the environment periodically and then transmit sensed data to the base station. Whereas on the other side, data transmitted by sensors are gathered at base station. A scenario is presented to the users depicting discovering of the occurred phenomenon. The network model incorporates some attributes:

- The nodes in the network model encompasses similar potential in terms of discovering, organizing and computing

- The nodes in the network model are energy driven;

\subsection{Radio Energy Model}

Radio energy model creates the estimation of energy employment [1]. As this model comprises of three modules namely transmitter, amplifier and receiver as shown in Figure.5 which indicates that the transmitter exhaust energy to transmit the information and receiver exhaust the energy for employing receiver with purpose of information reception. Radio energy model is depicted below in figure 5: 
This model comprises of two channel models free space model and multipath fading model, in which the over-reliance is deployed in accordance with extension within transmitter and receiver. In free space model The propagation depreciation of broadcasting power is designed as inversely proportional to $\mathrm{d}^{2}$ in free space model, where $\mathrm{d}$ is the distance between the transmitter and the receiver as well as the propagation depreciation of broadcasting power is designed as inversely proportional to $\mathrm{d}^{4}$ in multi path fading model. The amplifier is used for amplifying the broadcasting power. Thus the energy consumption for $\mathrm{K}$ bit data from transmitter to receiver at extension of $\mathrm{d}$ is defined as:

$$
\begin{aligned}
& \mathrm{E}_{\mathrm{Tx}}(\mathrm{L}, \mathrm{d})=\mathrm{L} * \mathrm{E}_{\text {elec }}+\mathrm{L} * \text { efs } * \mathrm{~d}^{2} \text { Where } \mathrm{d}<\mathrm{d}_{0} \\
& \mathrm{E}_{\mathrm{Tx}}(\mathrm{L}, \mathrm{d})=\mathrm{L} * \mathrm{E}_{\text {elec }}+\mathrm{L} * \text { empf } * \mathrm{~d}^{4} \text { Where } \mathrm{d}>=\mathrm{d}_{0}
\end{aligned}
$$

Th e energy consumption generated by the receiver for reception is:

$$
\mathrm{E}_{\mathrm{Rx}}(\mathrm{L}, \mathrm{d})=\mathrm{L} * \mathrm{E}_{\text {elec }}
$$

Where Eele represents the energy dissipated per bit to run the circuit. efs and empf stands for amplifier parameters used for free space channel model as well as multi path fading channel model respectively and do represent the crossover distance, and can be obtained from:

$$
\text { do }=\sqrt{\frac{E f s}{E a m p}}
$$

If the distance $\mathrm{d}$ is larger as compared to do, then multipath fading model is utilized, otherwise the free space model is present which is examined to estimate the energy employment in other respects.

\section{Related Work}

Authors in [8] proposed "Energy efficient and cluster based routing protocol for WSN". The base of this assignment is LEACH protocol (Low Energy Adaptive Clustering Hierarchy) which is the first and a classical hierarchical routing protocol in WSN. It initializes by dividing network into different clusters. First step includes the election of the cluster head. Second step involves data transmission between sensor nodes of the network and final transmission of data to the sink or base station. The consumption of the energy in network has been reduced greatly and its lifetime has been significantly improved.

Authors in [10] designed "Energy Efficient Routing in Wireless Sensor Networks through Balanced Clustering". The basis for the performance in this paper indulges LEACH protocol and it utilizes TDMA scheme for transmission of data and its sharing. In this paper, Gaussian elimination algorithm has been employed for minimizing the consumption of energy. The results represents the contraction in the energy consumption, thus the span of network has been improved significantly.

Authors in [9] determined "A Kind of Energy-efficient Routing Algorithm for WSN Based on HQEA". Authors present a hybrid QEA-based routing algorithm which is energy efficient represented by HERA in this paper. This conclusion is a combination of LEACH and PEGASIS., This algorithm uses the hybrid quantum evolutionary algorithm (HQEA) to minimize the distance between sender and receiver and in order to select the best cluster based multi-chain topology. Node's maximum energy along with the extension from the target is considered for the decrement in the consumption of energy and as a. result that energy consumption has been reduced as compared to the earlier results.

Authors in [11] proposed "Energy-Aware Routing in Wireless Sensor Networks Using Local Betweennesss Centrality", it introduces an energy aware dynamic routing strategy in WSN to improve the network lifetime that provides consumption of balanced energy. In this paper author proposes the Routing algorithm which uses a local betweenness centrality for estimation of the energy utilization by nearby nodes around a given local sensing node. It does not require any information about the architecture of sensor network or energy utilized by the entire network. If there occurs the situation like high traffic it performs the functions like congestion control and load balancing. Its centrality consumes energy more quickly as the distance between the nodes is large and the lifetime of the network can be increased by reworking the energy dissipation among nodes with the smaller local betweenness centrality.

Authors in [16] designed "A homogenous WSN algorithm: An energy aware cluster based approach" It comprises of three phases

1. Network levelling phase in which initialization takes place and nodes are placed in region of network.

2. Clustering phase, in this phase $\mathrm{CH}$ election take place and clusters are created.

3. Data transmission phase in which communication takes place between nodes.

Authors in[12] in 2015 designed "A decentralized energy efficient hierarchical cluster based routing algorithm for wireless sensor networks". In WSN, each sensor perform local processing and informs about the occurred events The data is transmitted to the base station either directly or by some intermediate nodes but also causes loss of coverage area and enhances time for occurred phenomenon diagnosis.

This approach in based on the concept in which initially the entire network is divided into clusters, each $\mathrm{CH}$ manages the cluster and assures energy dissipation.

The energy which is required for transmission of " $\mathrm{L}$ bit data" is given as

$\mathrm{E}_{\mathrm{t}}(\mathrm{L}, \mathrm{d})=\mathrm{L} * \mathrm{E}_{\text {elec }}+\mathrm{L} *$ efs $* \mathrm{~d}^{2} \quad$ Where $\mathrm{d}<\mathrm{d}_{0}$

$\mathrm{E}_{\mathrm{t}}(\mathrm{L}, \mathrm{d})=\mathrm{L} * \mathrm{E}_{\text {elec }}+\mathrm{L} *$ empf $* \mathrm{~d}^{4} \quad$ Where $\mathrm{d}>=\mathrm{d}_{0}$

Where $d$ represent transmission distance, Eelec stands for digital electronics whereas efs and empf represents the energy factors of amplifier for free space channell as well as multi path fading channel models respectively. do represent threshold distance which depends on the environment.

The energy for data reception by sensor node is given by

$$
\mathrm{E}_{\mathrm{r}}(\mathrm{L})=\mathrm{L} * \mathrm{E}_{\text {elec }}
$$

Their report show that the energy consumption has been reduced stretching network lifetime significantly.

Authors in [13] elucidates an "Energy Efficient Cluster Based Routing Protocol (EECBRP) for wireless sensor networks. Through network continuance and energy efficiency it successfully achieves in attaining alleviate performance. Sensor nodes are scattered it agglomerate data from sensors and transmit it to the base station. The architecture of EECBRP is designed to assist the remote monitoring in the sensor network periodically. In this paper electing cluster head $(\mathrm{CH})$, cluster scheme formation and a routing algorithm for carrying out data transmission between cluster head $(\mathrm{CH})$ and base station are major issues. Messages are exchanged between nodes and $\mathrm{CH}$ after that Cluster formation takes place. Cluster Head $(\mathrm{CH})$ election is rotated at a predefined interval for ensuring that energy is evenly distributed in the network which is the key function. The routing comprises of immaculate hauling data transmittal amidst cluster head $(\mathrm{CH})$ and sink by using multi-hop transmission. It assures that lifetime has been increased by $20 \%$ as compared to LEACH protocol. The result reports that energy consumption has been reduced.

Authors in [14] proposed "An Energy Efficient Hierarchical Clustering Technique for Wireless Sensor Network", in case for the purpose obtaining time critical data and periodic information from any area which comprises of distinct features through an energy efficient network. This paper comprises of two different protocols LEACH and TEEN These two protocols are incorporated together in case to monitor the time critical data and 
regular information, as there is demand for monitoring both of them in geographical areas. In order to avoid inter-cluster interface each cluster is assigned with DSSS spreading code. In case to avoid intra-cluster interference TDMA is used. Fused data which is received at $\mathrm{BS}$ from $\mathrm{CH}$ is avoided from collision by using MAC protocol. Simulation results report the increment in the lifetime of the network to a significant extent.

Nagendra Sah in [15] proposed "Performance Evaluation of Energy Efficient Routing in Wireless Sensor Networks". This paper aims for providing clustering method and cluster head selection method to improve energy efficiency wireless sensor network environment. This paper highlights comparison between LEACH and R-LEACH on the basis of its network lifetime. This paper gives description of work which shows modification of LEACH by introduction of cluster head $(\mathrm{CH})$ replacement scheme in an efficient way and inter-cluster transmission between the CH's. It gives the threshold in $\mathrm{CH}$ formation for very next round. If not much energy is spend by a $\mathrm{CH}$ during its round and the remaining energy is still more than the required threshold then the existing $\mathrm{CH}$ will be the $\mathrm{CH}$ again for the very next round. In this way energy consumption is carried out by saving the energy which is wasted in the form of routing packet for new $\mathrm{CH}$ election and hence R-LEACH provide better performance in spite of LEACH

\section{Conclusion}

As there is the scarcity in the energy resources of sensors, therefore energy adequacy becomes a growing challenge while constructing architecture of routing protocols in WSN. The dominating factors for energy utilization in sensor nodes include data transmission and data reception. The main objective behind the designing of protocols is keeping the sensors operating for a longer period of time, thus extending the network lifetime. Survey and summarization of modernistic research work comprises of hierarchical cluster-based routing protocols for wireless sensor networks is the main concern for the paper. As this is a very vast area, this paper has covered up only few samples of the routing protocols. In this paper the protocols which are altercated have their individual advantages and deficiencies. On the basis of topology, the protocol and different routing strategies can be applied.

\section{References}

[1] W. R. Heinzelman, A. P. Chandrakasan, and H. Balakrishnan, "Energy- efficient communication protocol for wireless micro sensor networks," in Proceedings of the 33rd Hawaii International Conference on System Sciences (HICSS-33), January 2000.

[2] A.Manjeshwar and D.P. Agrawal , "TEEN: a protocol for enhanced efficiency in wireless sensor networks", in Proceedings of the 1st International Workshop on Parallel and Distributed Computing Issues in Wireless Network, April 2001

[3] I. Akyildiz, W. Su, Y. Sankarsubramaniam and E. Caynei, "A survey on sensor networks", IEEE Communications Magazine, vol. 40, no. 8, pp. 102-114, August 2002.

[4] Ding, P., Holliday, J., Celik,"A Distributed Energy Efficient Hierarchical Clustering for Wireless Sensor Network", in proceedings of IEEE International Conference on Distributed Computing on Sensor Systems, Marina Del Rey, CA, June 2005.

[5] A.Manjeshwar and D.P. Agrawal, "APTEEN: a hybrid protocol for efficient routing and comprehensive information retrieval in wireless sensor networks", in Proceedings of the 2nd International Workshop on Parallel and Distributed Computing Issues in Wireless Networks and Mobile computing, Ft. Lauderdale, FL, April 2002.

[6] O. Younis and S. Fahmy, HEED: A hybrid, energy-efficient, distributed clustering approach for Ad Hoc sensor networks, IEEE Transactions on Mobile Computing, 3(4), 366-379,2004

[7] S. Lindsey, C. Raghavendra, "PEGASIS: Power Efficient Gathering in Sensor Information Systems," IEEE Aerospace Conference Proceedings, Vol. 3. No. 9- 16, 2002.
[8] Zhao, Zhou and Gao "Energy Efficient and Cluster Based Routing Protocol for WSN", IEEE, 2012.

[9] Lingxia Liu and Qiang Song, " A Kind of EnergyEfficient Routing Algorithm for WSN Based on HQEA", International Journal of Hybrid Information Technology, vol.6, No.4, 2013, pp.1-10.

[10] Stefanos A. Nikolidakis 1,*, Dionisis Kandris 2, Dimitrios D. Vergados 1 and Christos Douligeris, "Energy Efficient Routing in Wireless Sensor Networks Through Balanced Clustering”, Algorithms, Vol.6, 2013, pp.29-42.

[11] Xiao-Hui Li and Zhi-Hong Guan, "Energy- Aware Routing in Wireless Sensor Networks Using Local Betweenness Centrality", International Journal of Distributed Sensor Networks, 2013, pp.1-9.

[12] Maryam and Reza "A Decentralized Energy Efficient Hierarchical Cluster Based Routing Algorithm for Wireless Sensor Network", Elsevier International Journal of Electronics and Communications, 2015 [1434 - 8411].

[13] Krishna Chennakesava Rao.M Maheswar Vissa, Mrudula S, Ashutosh Kumar Dikshit"Energy efficient cluster based routing protocol for wireless sensor network", IEEE conference on ICCICCT, pp. 813-817, 2015.

[14] Kaushlendra Kumar Pandey, Bhagyashree Saud, Babli Kumari and Sonali Biswas"An energy efficient hierarchical clustering technique for wireless sensor network", IEEE conference publication, pp. 544-549, 2016.

[15] Nagendra Sah, " Performance evaluation of energy efficient routing in wireless sensor network", International Conference on Signal Processing Communication, Power and Embedded System, pp. 1048-1052,2016.

[16] Abolfazli and Mahdavi "A Homogeneous Wireless Sensor Network Routing Algorithm: An Energy Aware Cluster Based Approach” IEEE, 2014.

[17] Harikishore Kakarla, Madhavi Latha M and Habibulla Khan, "Transition Optimization in Fault Free Memory Application Using Bus-Align Mode", European Journal of Scientific Research, Vol.112, No.2, pp.237-245, ISSN: 1450216x135/1450-202x, October 2013.

[18] T. Padmapriya and V. Saminadan, "Improving Throughput for Downlink Multi user MIMO-LTE Advanced Networks using SINR approximation and Hierarchical CSI feedback", International Journal of Mobile Design Network and InnovationInderscience Publisher, ISSN : 1744-2850 vol. 6, no.1, pp. 14-23, May 2015.

[19] S.V.Manikanthan and K.srividhya "An Android based secure access control using ARM and cloud computing", Published in: Electronics and Communication Systems (ICECS), 2015 2nd International Conference on 26-27Feb.2015, Publisher: IEEEDOI:10.1109, /ECS.2015.7124833. 